\title{
Matching gaps between misaligned pointers demonstrates that lines, both transverse and parallel, enhance Poggendorff illusions
}

\author{
PETER WENDEROTH and TONY O'CONNOR \\ University of Sydney, Sydney, New South Wales, Australia
}

\begin{abstract}
Day and Kasperczyk (1985) and Day, Watson, and Jolly (1986) claimed that the Poggendorff illusion, in a Tolansky-type vertical bisection task, is no smaller when the line transversals are replaced with dots and the oblique parallels are removed, despite other evidence that transversals and parallels contribute significantly to the illusion. Wenderoth, O'Connor, and Johnson (1986) showed indirectly that both factors are important by instructing subjects to avoid a misunderstanding of the task, which was inferred to have occurred in the experiments of Day and his colleagues. In Experiment 1 of the present study, we demonstrated directly that this misunderstanding occurred when Day's instructions were used. In Experiment 2 we used a different, matching method for measuring standard Poggendorff effects, a method that avoids possible misinterpretations of the bisection task. In Experiment 3 we used this method to show directly that both line (vs. dot) transversals and oblique parallels (vs. no parallels) significantly increase illusions. The method of measurement used here should prove to be a useful tool in testing and placing constraints on theories of the Poggendorff effect.
\end{abstract}

A great deal of research on the Poggendorff illusion suggests that the apparent misalignment of the truly collinear transverse segments (which are vertical in Figure 1a) is greater when the illusory display is drawn with lines than when it is drawn with unconnected dots (e.g., Coren, 1970; Predebon, 1983; Wenderoth \& Wade, 1981). There also is considerable evidence that the illusion is greater when the inducing parallels are present than when they are absent (e.g.,. Curthoys, Wenderoth, \& Harris, 1975; Day, 1973; Goldstein \& Weintraub, 1972; Wundt, 1893).

It is quite clear, however, that factors other than angular distortion of abutting lines are involved in the illusion, because certain manipulations of the display do not affect the illusion's magnitude in a manner consistent with a simple explanation couched solely in terms of angular distortion (e.g., Day \& Dickinson, 1976; Tong \& Weintraub, 1974; Wenderoth, 1980, 1981b; Wenderoth \& Johnson, 1981). Nevertheless, it was somewhat surprising when Day and Kasperczyk (1985) reported that four variants of the Tolansky version of the illusion (Figures $1 \mathrm{~b}$ to $1 \mathrm{e}$ ) all induced effects of equal magnitude: in every case the upper pointer (line or dot) was set down to the right by the same incorrect amount in order to appear to vertically bisect the horizontal distance between the two lower targets. It was even more surprising when Day,

This research was supported by Grant A 28515620 I from the Australian Research Grants Scheme to the first author, which provided for the second author's research assistance. We thank John Holden for assistance with computing facilities and Dan Weintraub for invaluable constructive criticisms of the original manuscript. The authors' mailing address is: Department of Psychology, University of Sydney, Sydney, N.S.W., Australia 2006.

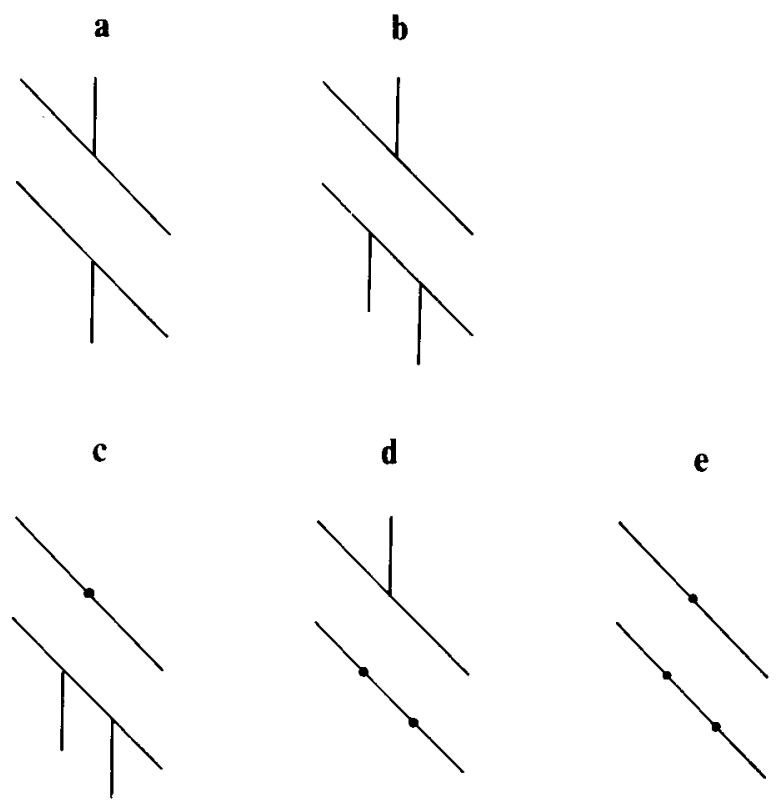

Figure 1. Variants of the Poggendorff illusion. (a) The conventional form but with transversals vertical and parallel endpoints alligned vertically. (b) A Tolansky variant. (c, d, e) Tolansky variants in which some or all transversals are replaced with dots.

Watson, and Jolly (1986) reported that identical illusions were induced by Figures $1 \mathrm{~b}$ and $1 \mathrm{e}$, and the parallel-less versions of these two figures. Such evidence would imply that neither the transverse line segments nor the parallels play any significant role in the Poggendorff effect, an implication quite contrary to previous research findings. 
Wenderoth, O'Connor, and Johnson (1986) suggested possible reasons for Day and Kasperczyk's (1985) results. Day and Kasperczyk instructed their subjects to "move the upper element along the diagonal parallel so that it lay exactly halfway between the two lower ones, that is, so that if it were to be extended vertically downward it would exactly bisect the space between the two lower elements" (p. 76). With these or similar instructions, subjects may have interpreted the task, especially the threedot task (Figure le), incorrectly. Specifically, some subjects may have set the upper dot so that it obliquely bisected the line joining the two lower dots, that is, so that it lay on the virtual line orthogonally bisecting the line joining the two lower dots. Consistent with this hypothesis was the fact that the standard deviation $(S D)$ of settings in Day and Kasperczyk's three-dot condition (2.06) was more than three times larger than that for the threeline condition (0.67).

To test this hypothesis indirectly, Wenderoth et al. (1986) instructed subjects not to confuse vertical and oblique bisection and not to attempt oblique bisection under any circumstances. Using these instructions and displays such as those in Figure 1 (b-e), both with and without parallels, Wenderoth et al. obtained the following results. First, adding parallels to the displays significantly increased illusory errors when the upper pointer was a line but notwhen it was a dot, although all obtained means with parallels exceeded those without. Second, although the variances of means for the all-dot displays exceeded those for the all-line displays, the variance inhomogeneity was not significant, as it had been in Day and Kasperczyk's (1985) study. Wenderoth et al. concluded that "displacement effects with dots on oblique parallels are not as great as those with lines on oblique parallels," and that "accordingly, explanations of the Poggendorff illusion which include some contribution of line and angle interactions remain tenable" (Wenderoth et al., 1986, p. 337).

Recently, Day et al. (1986) presented further evidence for their view that neither parallels nor line pointers contribute to the Poggendorff effect. They repeated Day and Kasperczyk's (1985) experiments, this time using Figures $1 \mathrm{~b}$ and le both with and without parallels. They found no significant differences among the mean errors for the four displays, but the pattern of results was identical to that in Day and Kasperczyk's study. First, when all subjects completed all conditions (repeated measures), the obtained dot-figure means exceeded the line-figure means, but the same was true of the $S D$ s. For displays with and without parallels, the dot-figure $S D$ s were 1.31 and 1.35 , respectively; the line-figure $S D$ s were 0.79 and 0.62 , respectively. When independent groups judged just one display each, the all-line display with parallels and the all-dot display without parallels, the $S D$ s were more comparable ( 0.86 and $0.64 \mathrm{~mm}$, respectively), but the obtained line-figure mean exceeded the dot-figure mean (1.17 vs. $0.88 \mathrm{~mm})$, although not significantly so.
Our primary aim in the present study was to attempt to demonstrate directly, using a different method, that alldot illusions are not equal in magnitude to all-line illusions. First, however, we decided to repeat (although not replicate) Day et al.'s (1986) Experiment 1, using the instructions used by Day et al. and by Day and Kasperczyk (1985), just to see whether some subjects would misinterpret the task as oblique bisection. Subjects were read the instructions at the beginning of the experiment and were quizzed afterward as to whether they had or had not misunderstood, or whether, at least, they had attempted the incorrect bisection on any trials.

There was one substantial difference between our displays and those used by Day et al. (1986). Day et al. used oblique parallels truncated so that their endpoints were vertical (as in Figure 1), whereas we used parallels whose endpoints were obliquely aligned (as in Figure 4). There were three main reasons for this decision. First, experience had taught us that our subjects, given the opportunity, would rapidly develop strategies to achieve correct judgments by other than the required means. With the parallels arranged as in Figure 1, subjects could easily achieve the correct setting by setting the upper pointer exactly to bisect the upper parallel line without ever attending to the lower parallel or the lower targets. We preferred to avoid this possibility. Second, Day (1974) had shown that when one line is bisected by another, nonorthogonal line, the half of the bisected line on the acute angle side appears shorter than the half on the obtuse angle side. Hence, if subjects adopted the bisection strategy, this alone would produce errors in the Poggendorff direction, a confounding best avoided. Third, Gillam (1971) and Brigell and Uhlarik (1980) had shown that the standard (single transversal) Poggendorff illusion is near zero when the parallels are vertical and their endpoints are aligned parallel to the transversal (i.e., Figure 1a rotated $45^{\circ}$ clockwise). Even though this reduction may be due to perspective cues that operate only when the parallels are vertical (Wenderoth, 1981a), we wanted to avoid using any display involving extraneous variables in examining an issue as fundamental as the role of oriented line segments and parallel inducing lines in the production of the Poggendorff effect.

For these reasons, we preferred to use the rotated version of the classic display in Experiment 1; in Experiments 2 and 3 we employed a different method of measurement for which the manner of parallel truncation is largely irrelevant.

\section{EXPERIMENT 1}

\section{Method}

Apparatus. Stimulus displays were presented on the flat face of a Tektronix 608 monitor (P4 phosphor) stationed $57 \mathrm{~cm}$ from the subject's padded head- and chinrest so that $1 \mathrm{~cm}$ on the screen subtended $1^{\circ}$ of visual angle. The subject had control of three microswitches, the outer two or which could move the adjustable 
stimulus left or right along its oblique track. The display screen was interfaced with a Hewlett-Packard 1350A graphics translator and a PDP-11/20 computer, which recorded all settings. When the subject pressed the center switch, the response was stored, the current display was erased, and the next stimulus appeared.

Stimuli. Four stimulus displays were used; two were similar to those shown in Figures $1 \mathrm{~b}$ and le (which are not drawn to scale) and two were the same displays minus the parallels. The oblique parallels, when present, were $80 \mathrm{~mm}\left(8^{\circ}\right)$ long and were separated by $15 \mathrm{~mm}$. All lines were $10 \mathrm{~mm}$ long and the oblique separation between the two lower lines (or dots) was $10 \mathrm{~mm}$.

One tap on one of the outer microswitches moved the upper line or dot $0.14 \mathrm{~mm}$. Continuous pressing produced apparently smooth movement, at rates of approximately $0.9 \mathrm{~mm} / \mathrm{sec}$ for lines and $0.25 \mathrm{~mm} / \mathrm{sec}$ for dots (see Wenderoth et al., 1986). The luminance of all lines was approximately $1.9 \mathrm{~cd} / \mathrm{m}^{2}$ and contrast, defined as $\left(L_{\max }-L_{\min }\right) /\left(L_{\max }+L_{\min }\right)$, was about 1.0 , because background luminance was too low to be measurable (SEI photometer).

Procedure. The subjects were briefly shown drawings resembling Figures $1 \mathrm{~b}$ and $1 \mathrm{e}$ and their parallel-less counterparts. They were then read the following:

Your task in this experiment is to move the upper element along the diagonal parallel so that it lies exactly halfway between the two lower ones, that is, so that if it were to be extended vertically downward it would exactly bisect the space between the two lower elements. OK?

If any subject queried the instructions or asked for extra information, the experimenter simply reread the original instructions. No additional clarification was given.
Each subject made 16 settings, 4 for each display in random order, from starting positions \pm 2.5 and $\pm 5 \mathrm{~mm}$ from physical vertical bisection. Errors were measured in millimeters along the upper parallel with errors down and to the right called positive, so that true vertical bisection would be a zero error and (incorrect) oblique bisection would be a $15-\mathrm{mm}$ error.

Subjects. There were 15 subjects, volunteers from an introductory psychology course, all with emmetropic (normal) or corrected vision.

\section{Results}

Of the 15 subjects, 6 admitted quite readily to using the incorrect oblique bisection response on at least some trials. Inspection of individual data confirmed this: some of these 6 subjects made errors of $10 \mathrm{~mm}$ or more $(15 \mathrm{~mm}$ $=$ physical oblique bisection) on most of the eight trials with dot stimuli; others did so on only one or two. Using the formula $\alpha^{\circ}=45^{\circ}-\tan ^{-1}$ [(15-x)/15], where $x=$ error in millimeters and 15 represents the interparallel distance, mean errors (in millimeters) were converted to angular errors (Wenderoth, Beh, \& White, 1978) for the total sample and separately for the 6 subjects who admitted to using oblique bisection (Group 0 ) and the 9 who did not (Group V). The mean errors in millimeters are shown in Figure 2.

Complete statistical analyses were not carried out on these data, first, because the total sample clearly was inhomogeneous; second, because the two subsamples $(n=6$

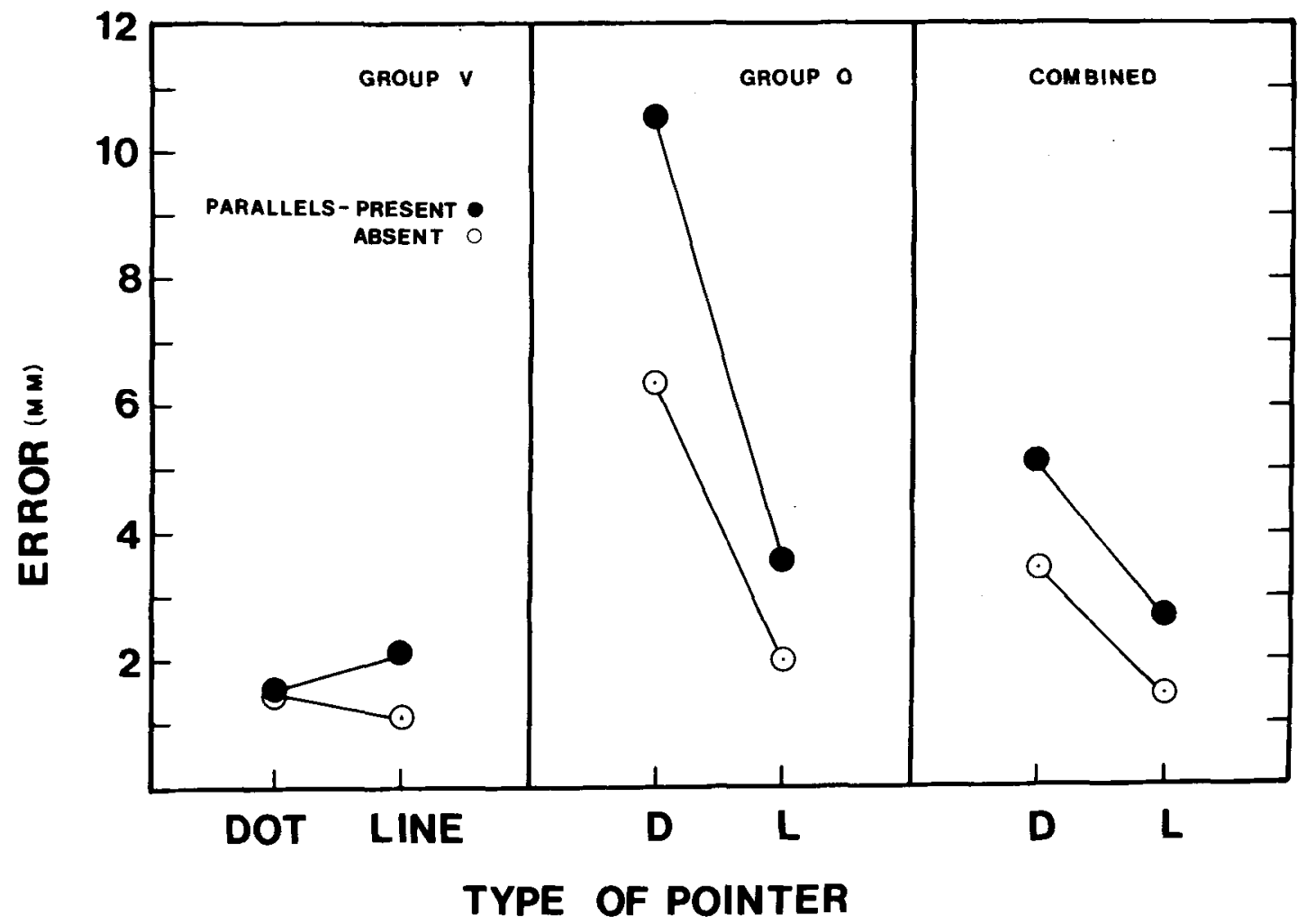

Figure 2. Mean bisection errors, in millimeters, obtained in Experiment 1 with dot and line pointers. Left panel: Results for Group V subjects, who claimed not to have attempted oblique bisection $(n=9)$. Center panel: Results for Group $O$ subjects, who admitted to using oblique bisection $(n=6)$. Right panel: Combined results $(n=15)$. 
and $n=9$ ) were very small; and third, because the results were so dramatic. However, tests were conducted on the means of Group V, in which the variances were at least reasonably homogeneous: Figure 3 shows the standard deviations associated wtih the means (in millimeters) in Figure 2.

Using the errors in millimeters, we conducted a simple repeated measures analysis of variance with three planned contrasts (Winer, 1962, chap. 4). First, in the left panel of Figure 2, the mean errors for dot settings without and with parallels were 1.45 and $1.54 \mathrm{~mm}\left(2.91^{\circ}\right.$ and $3.10^{\circ}$ ), respectively. This difference was not significant $[F(1,24)=0.09, p>.05]$. However, the mean errors for line settings without and with parallels were 1.09 and $2.11 \mathrm{~mm}\left(2.16^{\circ}\right.$ and $\left.4.33^{\circ}\right)$, and this difference was significant $[F(1,24)=11.07, p<.005]$. The mean of all dot-setting errors $(1.495 \mathrm{~mm})$ did not differ from the mean of all line-setting errors $(1.60 \mathrm{~mm})[F(1,24)=0.23$, $p>.05]$.

\section{Discussion}

The results of Experiment 1 demonstrate unequivocally that unless extreme care is taken, at least some subjects undertaking a vertical bisection task of the Tolansky type will misinterpret the instructions on at least some trials and will complete an incorrect oblique bisection task. This experiment completely confirms the earlier data and hypotheses of Wenderoth et al. (1986). First, even for subjects in Group V, who claimed not to have misinterpreted the instructions, the dot-setting $S D$ s were slightly larger than those for lines (Figure 3, left panel). Wenderoth et al. obtained the same result in subjects instructed to avoid oblique bisection: the dot-setting task was less precisely performed. Second, Wenderoth et al. proposed that incorrect oblique bisection would be less likely to occur with line stimuli because the lines partially define the direction of bisection. The means in Figure 2 (center panel) are consistent with this idea: for Group 0 , the mean setting errors for lines without and with parallels were 2.00 and $3.57 \mathrm{~mm}$, respectively, but the dot-setting errors were 6.36 and $10.55 \mathrm{~mm}$ (true oblique bisection is

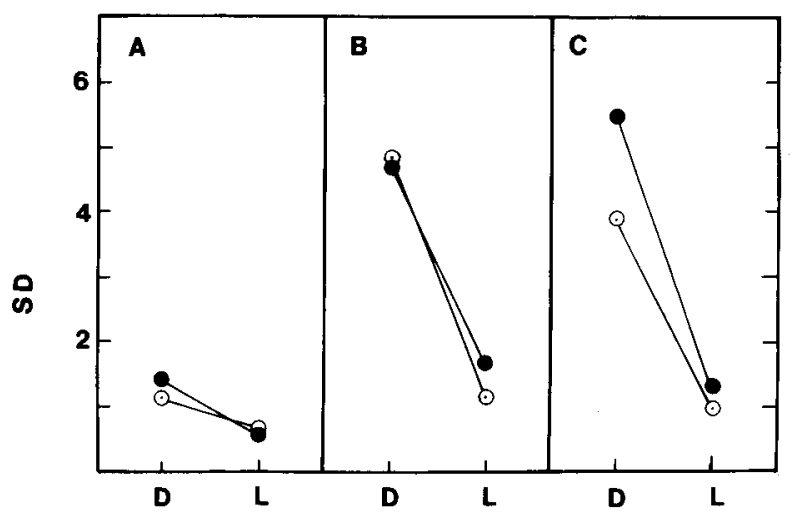

Figure 3. Standard deviations of mean bisection errors shown in Figure 1.

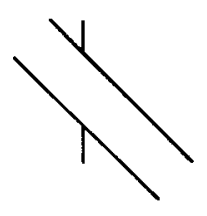

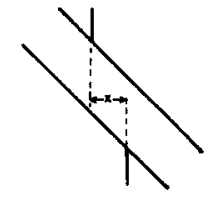

b $\because 0$

6
Figure 4. Type of display and judgmental task used in Experiment 2. (a) Poggendorfi figure with transverse segments collinear. (b) Transverse segments misaligned by amount $x$. (c) Pair of dots used by subject to estimate distance $x$. Arrows indicate that dots moved in or out together.

$15 \mathrm{~mm}$ ). Because the range of possible settings is so great, especially for dots in Group $\mathrm{O}$, the variances of dot-setting errors in this group, and hence in the total sample, were very large (Figure 3, center and right panels).

Finally, Wenderoth et al. (1986) proposed that the addition of oblique parallels to the dot displays "increases the salience of the oblique bisection alternative and biases subjects' settings in that direction" (Wenderoth et al., 1986, p. 337). The apparent interaction shown in Figure 2 (center panel) is consistent with this idea: the addition of parallels increased line-setting errors by only $1.57 \mathrm{~mm}$ $\left(3.6^{\circ}\right)$, but increased dot-setting errors by $3.19 \mathrm{~mm}$ $\left(13.41^{\circ}\right)$.

From the previous research of Wenderoth et al. (1986) and from the statistical tests carried out on the data of Group V (Figure 2, left panel), we conclude again that line interactions are a significant component of the Poggendorff illusion and that both the line pointers and the parallels contribute significantly to the effect.

\section{EXPERIMENT 2}

Our aim in Experiment 2 was to use a different method of measuring the Poggendorff illusion, for two reasons. First, if this method worked, we could use it to compare illusions obtained with line and dot pointers in nonTolansky variants of the illusion, thus avoiding altogether the problem of misunderstood instructions. Second, the method seemed to have important implications for various theories of the illusion.

Consider the method first. Figure 4a shows a Poggendorff figure in which the truly collinear vertical segments appear to be misaligned. In Figure 4b, the segments actually are misaligned. The subjects were presented with displays similar to Figure $4 b$, with variou amounts of physical horizontal misalignment between the vertical lines. Their task was to vary the separation of a pair of dots (Figure 4c) to match the horizontal misalignment in the Poggendorff figure.

The test of whether this method could be used to measure the illusion was whether similar amounts of illusion would occur independently of the actual amount of separation between the lines. If this were the case, it would be possible then to ask subjects to set the dot separation to match the separation of line pointers and also of dot 
pointers to establish whether line pointers induced larger effects.

This experiment is theoretically important, too, because some theories of the Poggendorff illusion attribute at least part of the effect to a stretching of perceptual space within the parallels (see Day \& Kasperczyk, 1985). Other theories attribute the illusion to angular distortions such that the transverse lines appear not to point at each other (e.g., Wenderoth et al., 1978). Clearly, if the illusion is due to a pivoting of the transverse lines about their points of intersection with the parallels, the method described in relation to Figure 4 should reveal a substantial illusion only when the lines truly are collinear. In cases where there is a physical separation, any apparent bending or change in apparent orientation of the lines should not greatly affect the judged horizontal separation of their points of intersection.

The method we developed to measure the illusion is a variant of methods used by others. Restle (1969) presented truly misaligned transversals and had subjects rate the degree of misalignment, whereas Krantz and Weintraub (1973) asked subjects to set a pair of dots to match the perceived misalignment along the inducing parallel. Our method differs from the method of Krantz and Weintraub in that they required subjects to imagine where the virtual extension of one transversal would intersect the distal parallel, and then to estimate the distance from this point to the actual target. We asked observers merely to match the horizontal separation between the endpoints of the transversals. We also took pretest measures of matched separation in the absence of inducing parallels.

\section{Method}

Apparatus. The apparatus was the same as that used in Experiment 1 , except that the outer microswitches now caused the matching dots to separate or to converge horizontally, in synergistic fashion, at a rate of $0.25 \mathrm{~mm} / \mathrm{sec}$.

Stimuli. The oblique parallels were $50 \mathrm{~mm}\left(5^{\circ}\right)$ long, and were separated by $10 \mathrm{~mm}$, so that the vertical distance between the parallels was $14.14 \mathrm{~mm}$. When the 10 -mm-long transverse segments truly were aligned, the lower segment intersected the lower parallel at its center. There were four other stimuli, in which the upper line segment was displaced obliquely (along the upper parallel) by either -1.5 or $-3.0 \mathrm{~mm}$ (up and to the left) or by +1.5 or $+3.0 \mathrm{~mm}$ (down and to the right). When vertically aligned, the pair of matching dots were $20 \mathrm{~mm}\left(2^{\circ}\right)$ to the right of the parallels, measured horizontally from the point $7.07 \mathrm{~mm}$ above the lower line segment's point of abuttal with the lower parallel. The dots were separated vertically by $3 \mathrm{~mm}\left(3^{\circ}\right)$, so that the upper dot clearly referred to the upper line tip and subjects did not confuse negative separations (upper line to the left) with positive ones (upper line to the right).

Procedure. The subjects were instructed to set the dot separation to match the apparent horizontal distance between the two vertical line segments. Every subject judged each of the 10 displays (5 separations with and without parallels) four times each, from starting positions $\pm 2.5 \mathrm{~mm}$ and $\pm 5 \mathrm{~mm}$ from true vertical alignment. The $\mathbf{4 0}$ trials were in a different random order for each subject.

Subjects. There were 22 subjects, taken from the same population as those in Experiment 1.

\section{Results}

The mean matching errors obtained without parallels (open circles) and with parallels (filled circles) are shown in Figure 5, with an error bar for \pm 1 standard error. The graph plots judged oblique separation against true oblique separation: the obtained horizontal estimates $(h)$ were transformed to estimates of oblique (o) separation by $o$ $=\sqrt{2 h^{2}}$, as was the standard error.

The most noticeable feature of Figure 5 is that the errors with the parallels present show a uniform Poggendorff error relative to true collinearity (dashed line) regardless of the extent of true misalignment, whereas the errors when parallels were absent regress toward the mean misalignment (i.e., zero). This is illustrated by the fact that the best-fit regression line to the errors with parallels present has a slope of 0.98 , whereas the regression line to the errors with parallels absent has a slope much less than 1 , namely 0.84 . We have no explanation of this regression to the mean, but we note that it has not occurred in other experiments conducted after this one, so there is little point in dwelling upon it. Both Restle (1969) and Krantz and Weintraub (1973) obtained with-parallels regression coefficients close to unit, so this aspect of our data confirms those results.

Using a simple subjects $\times$ treatments design with planned contrasts, tests confirmed the differences between the regression coefficients. Thus, the differences between the with- and without-parallels mean errors were significantly different for true misalignments of $-3,-1.5$, and $0 \mathrm{~mm}[F(1,189)=9.46,6.49$, and 5.89 , respectively, $p<.005, p<.025$, and $p<.025]$, whereas the differences for separations of +1.5 and $+3 \mathrm{~mm}$ were not significant $[F(1,189)=2.93$ and $0.93, p>.05$ in both cases]. Overall, the combined with- and without-parallels data exhibited a significant linear trend $(F(1,189)=$ $409.64, p<.0005)$, but not a quadratic $(F=0.86)$, cubic $(F=1.80)$, or quartic trend $(F=0.07)$.

The overall mean difference between the with- and without-parallels horizontal judgments was $0.642 \mathrm{~mm}$, which translates into a transformed oblique difference in Figure 5 of $0.91 \mathrm{~mm}$ (the difference between the intercepts of the regression lines). Although this difference may seem small, it is consistent with previous research. Thus, it is well established that, in angular terms, the Poggendorff illusion is between about .25 and .4 as large when the transversals are vertical as when they are oblique, $6^{\circ}$ $8^{\circ}$ in the latter case and $1^{\circ}-2^{\circ}$ in the former case (Leibowitz \& Toffey, 1966; Weintraub \& Krantz, 1971; Weintraub, Krantz, \& Olson, 1980). Using the formula given earlier, the $0.91-\mathrm{mm}$ effect obtained here translates to an angular error of $1.90^{\circ}$. This is consistent, too, with the well-established relationship between the illusion magnitude $(M)$, the acute angle between transversals and parallels $(A)$, and the width between the parallels $(W)$, namely $M=0.162 W \cot A$ (Weintraub \& Krantz, 1971). This formula predicts an illusion here of $0.162(15.1)=$ 


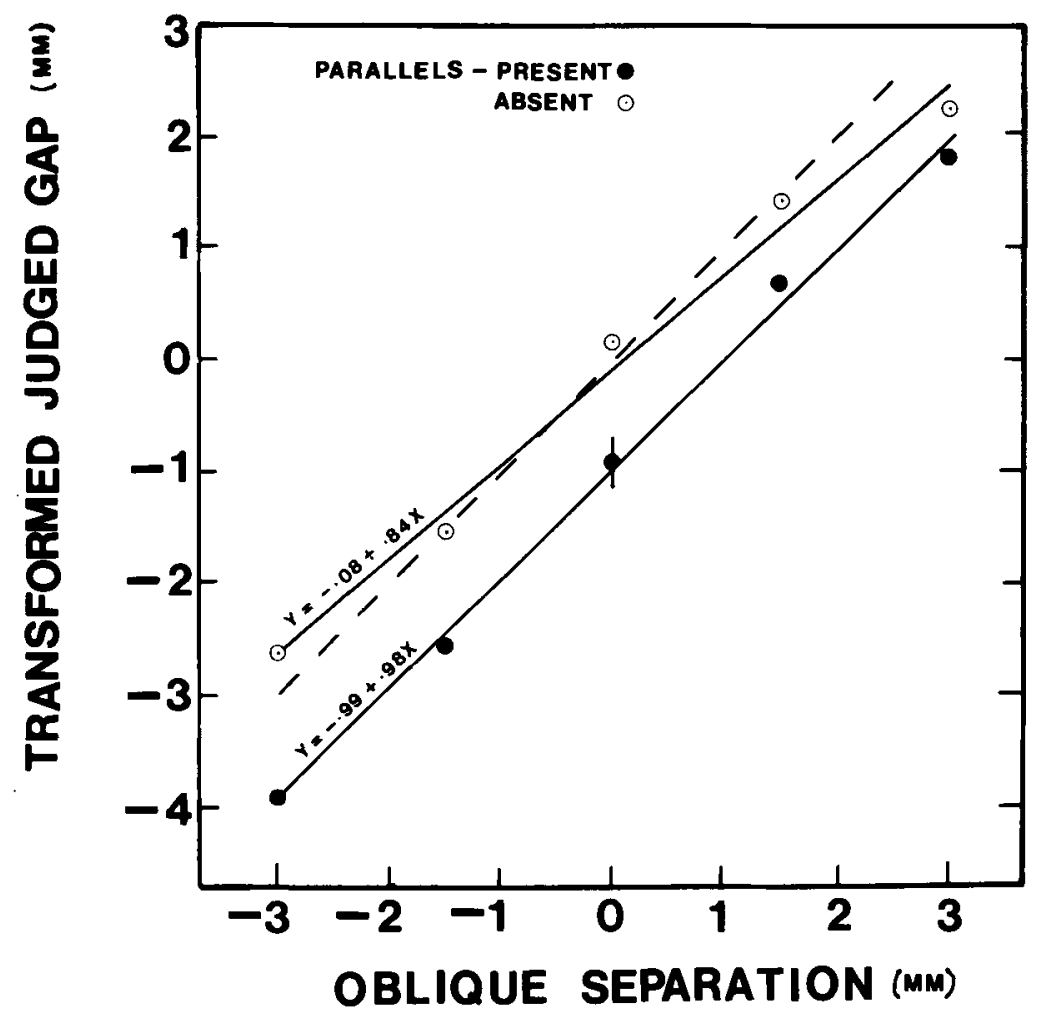

Figure 5. Estimated judged oblique separation of transverse lines as a function of actual oblique separation, with parallels present (solid symbols) and absent (open symbols). Subjects judged horizontal extent $(h)$, which was transformed to estimated oblique extent by $\sqrt{2 h^{2}}$. Dashed line shows zero error (perfect judgment). Best fit regression lines are shown by solid lines.

$2.43 \mathrm{~mm}$ with upright parallels, and between $0.25 \times 2.43 \mathrm{~mm}(0.61 \mathrm{~mm})$ and $0.40 \times 2.43 \mathrm{~mm}$ $(0.97 \mathrm{~mm})$ with oblique parallels.

\section{Discussion}

This experiment indicates that the Poggendorff illusion persists when there is a physical separation between the transverse line segments, a finding that is consistent with the findings of Restle (1969) and Krantz and Weintraub (1973). Although this has implications for certain theories of the effect (e.g., it seems to falsify those that rely upon angular rotation of the transversals about their points of intersection), our primary purpose here was to establish that effects can be reliably measured with noncollinear vertical line segments, using our matching technique.

\section{EXPERIMENT 3}

Armed with the technique of measuring Poggendorff effects that was used in Experiment 2, we designed Experiment 3 directly to compare illusions induced by dot and line transversals. The transversal separation was always one of the five used in Experiment 2, namely, the $-1.5-\mathrm{mm}$ separation in which pretest judgments were close to veridical, although other trials, with random sizes of positive separation, were randomly interspersed so that the subject would be unaware that the physical separation of interest was constant.

\section{Method}

Apparatus and Procedure. The apparatus and procedure were the same as those used in Experiment 2.

Stimuli. The stimuli with line transversals were identical to those used in Experiment 2 for the -1.5 -mm oblique separation, one with parallels and one without. The two other displays were identical except that the line segments were replaced by dots on the parallels.

Subjects. The 12 subjects were volunteers taken from the same population as the subjects in Experiments 1 and 2.

\section{Results}

The mean errors, in millimeters, obtained without parallels were $-0.61 \mathrm{~mm}$ for both line and dot transversals. Adding the parallels increased the dot illusion by $0.59 \mathrm{~mm}$ and the line illusion by $0.99 \mathrm{~mm}$. With a very small overall standard error $(0.121)$, there were significant differences.

One contrast showed that the parallel-less effects were the same (the means were identical, so $F=0$ ). A second contrast tested the difference between the with-parallels line-transversal mean error $(-1.60 \mathrm{~mm})$ and the withparallels dot-transversal mean error $(-1.20 \mathrm{~mm})$. This 
difference was significant $[F(1,33)=5.78, p<.025]$. Finally, the with-parallels overall mean $(-1.40 \mathrm{~mm})$ differed from the parallel-less overall mean $(-0.61 \mathrm{~mm})$ $[F(1,33)=30.08, p<.0005]$.

\section{Discussion}

Experiment 3 demonstrated in a new way that adding parallels to Poggendorff transversals increases the effect significantly, and that when parallels are present, transverse line segments result in larger illusions than those that occur with dots. This confirms and extends the earlier findings of Wenderoth et al. (1986).

\section{GENERAL DISCUSSION}

Experiment 1 showed directly that some subjects given the instructions used by Day and Kasperczyk (1985) and Day et al. (1986) in relation to a Tolansky-type vertical bisection task tended to misinterpret the instructions and to carry out an inappropriate oblique bisection. We can infer that such inappropriate settings were the major reason for Day et al.'s (1986) conclusion that neither line transversal segments (compared to dots) nor oblique parallels contribute anything to the Poggendorff illusion. The present findings are consistent with those obtained by Wenderoth et al. (1986), who came to the same conclusion by different means. After specifically instructing subjects not to attempt oblique bisection, they obtained significantly greater illusions with line, rather than dot, transversals and with oblique, rather than no, parallels.

It is possible that the results of Experiment 1 are contingent upon our choice of obliquely oriented parallel endpoints. We consider that to be unlikely, partly because the parallels were so long, so that these endpoints were about $4^{\circ}$ peripheral to the targets. One might contemplate an experiment that would directly compare the results obtained with vertical endpoints and those obtained with oblique endpoints in independent samples, but such a study would have to avoid the possible confoundings discussed earlier. It is perhaps worth mentioning here that we have conducted further experiments in which the parallel lines of a Tolansky figure were horizontal with vertically aligned endpoints and with the transversal segments oblique. The subjects' task was to place the upper, single oblique transversal so that its point of intersection with the upper parallel lay directly above the midpoint between the points of intersection of the two lower oblique transversals. Here, too, subjects given a nonprohibitive instruction misinterpreted the task: 8 of 12 subjects admitted afterward to setting the upper transversal so that the whole upper transversal pointed to the lower midpoint. This error occurred only with line, not dot, transversals, and it occurred with lines whether parallels were present or not. A group given prohibitive instructions made no such errors. Here, then, the direction of the transversals (rather than the parallels) gave rise to a misinterpretation of the task. In any event, our primary aim in the present study was to confirm that the presence of both parallels and oriented transversals results in more potent illusory effects, and this was demonstrated convincingly in Experiment 3, independently of the Tolansky task and all of its associated complications.

Experiment 2 confirmed that the Poggendorff illusion with single transversal segments can be measured by physically misaligning the transversals and having observers match the separation with dots. This method could be a valuable tool in exploring the multiple determinants of the illusion, as noted by Krantz and Weintraub (1973).

For immediate purposes, however, this method enabled us to design Experiment 3 to test directly whether line transversals and oblique parallels contribute to the Poggendorff effect. They do: adding parallels increases the effect, and when parallels are present, larger effects occur with line transversals than with dots. Note that this last finding seems to negate a recent theory of the Poggendorff effect couched in terms of Müller-Lyer illusions (Greist-Bousquet \& Schiffman, 1981, 1985), because the Müller-Lyer components essential to that theory occur in the figure both when the transverse segments are lines and when they are dots.

\section{REFERENCES}

Brigell, M., \&hlarik, J. (1980). Bending the parallels of the Poggendorff figure. Bulletin of the Psychonomic Society, 16, 1-4.

COREN, S. (1970). Lateral inhibition and geometric illusions. Quarterly Joumal of Experimental Psychology, 22, 274-278.

Curthoys, I., Wenderoth, P., HaRRIS, J. (1975). The effects of the motion path and the length of the variable segment in the Poggendorff illusion without parallels. Perception \& Psychophysics, 17, 358-362.

DAy, R. H. (1973). The oblique line illusion: The Poggendorff effect without parallels. Quarterly Journal of Experimental Psychology, 25, 535-541.

D^y, R. H. (1974). The Poggendorff illusion: Apparent displacement of the oblique lines. Australian Journal of Psychology, 26, 49-59.

DAY, R. H., \& Dickinson, R. G. (1976). The components of the Poggendorff illusion. British Journal of Psychology, 67, 537-552.

DAY, R. H., KASPERCZYK, R. T. (1985). Apparent displacement of lines and dots in a parallel-line figure: A clue to the basis of the Poggendorff effect. Perception \& Psychophysics, 38, 74-80.

Day, R. H., Watson, W. L., Jolly, W. J. (1986). The Poggendorff displacement effect with only three dots. Perception \& Psychophysics, 39, 351-354.

Gillam, B. (1971). A depth processing theory of the Poggendorff illusion. Perception \& Psychophysics, 10, 211-216.

GoldsteIn, M. B., \& WeintraUB, D. J. (1972). The parallel-less Poggendorff: Virtual contours put the illusion down but not out. Perception \& Psychophysics, 11, 353-355.

Greist-Bousquet, S., SCHifman, H. R. (1981). The Poggendorff illusion: An illusion of linear extent? Perception, 10, 155-164.

Greist-Bousquet, S., \&CHIFFmaN, H. R. (1985). Poggendorff and Müller-Lyer illusions: Common effects. Perception, 14, 427-447.

KrantZ, D., \& Weintraub, D. (1973). Factors affecting perceived orientation of the Poggendorff transversal. Perception \& Psychophysics, 14, 511-517.

LeibowITz, H., \& ToFrey, S. (1966). The effects of rotation and tilt on the magnitude of the Poggendorff illusion. Vision Research, 6, 101-103.

Predebon, J. (1983). Recognition processes and the occurrence of the dot Poggendorff illusion. Perceptual \& Motor Skills, 36, 394. 
Restle, F. (1969). Illusions of a bent line. Perception \& Psychophysics, 5, 273-274.

Tong, L., * Weintraub, D. J. (1974). Contour displacements and tracking errors: Probing 'twixt Poggendorff parallels. Perception \& Psychophysics, 15, 258-268.

Weintraub, D. J., \& Krantz, D. H. (1971). The Poggendorff illusion: Amputations, rotations, and other perturbations. Perception \& Psychophysics, 10, 257-264.

Weintraub, D., Krantz, D. H., Olson, T. P. (1980). The Poggendorff illusion: Consider all the angles. Journal of Experimental Psychology: Human Perception \& Performance, 6, 718-725.

WENDEROTH, P. (1980). Alignment errors in Poggendorff-like displays when the variable segment is a dot, a dot series, or a line. Perception \& Psychophysics, 27, 505-518.

Wenderoth, P. (1981a). Bending the Poggendorff parallels and the rules of inference: A note on Brigell and Uhlarik (1980). Perception \& Psychophysics, 29, 283-284.

Wenderoth, P. (1981b). The role of the second oblique in the Poggendorff illusion. Perception, 10, 605-614.
Wenderoth, P., Beh, H., \& White, D. (1978). Perceptual distortion of an oblique line in the presence of an abutting vertical line. Vision Research, 18, 923-930.

Wenderoth, P., Johnson, M. (1981). Are the obtuse angles the key components of the Poggendorff illusion? Perception, 10, 165-172. WENDERoTh, P., O'ConNOR, T., \& Johnson, M. (1986). Evidence for a significant contribution of interactions between oriented line segments in the Tolansky version of the Poggendorff illusion. Perception \& Psychophysics, 39, 334-338.

Wenderoth, P., \& WADE, N. (1981). An investigation of line and dot forms of the Müller-Lyer and Poggendorff illusions. Quarterly Journal of Experimental Psychology, 33A, 77-85.

WINER, B. J. (1962). Statistical principles in experimental design. New York: McGraw-Hill.

WuNDT, W. (1893). Grundzüge der physiologischen Psychologie. Leipzig: Engelmann.

(Manuscript received May 28, 1986; revision accepted for publication November 4,1986 .) 